\title{
Optimal Investment Strategies in a CIR Framework.
}

\author{
Griselda Deelstra, CREST and ENSAE. \\ Martino Grasselli, CREST* \\ Pierre-François Koehl, CDC and DABF!
}

June 8, 2000

December 1998

Abstract: We study an optimal investment problem in a continuous-time framework where the interest rates follow the Cox-Ingersoll-Ross dynamics. Closed form formulae for the optimal investment strategy are obtained by assuming the completeness of financial markets and CRRA utility function. In particular, we study the behaviour of the solution when time approaches to the terminal date.

Key words: consumption-investment strategy, stochastic interest rates, stochastic optimization.

Classification according to JEL codes: G11

\section{Introduction}

We study an optimal investment problem with a stochastic term structure of the interest rates.

Optimal consumption-investment problems are studied in the literature since a long time (see e.g. Merton 1971 where the interest rates are constant).

\footnotetext{
${ }^{*}$ Corresponding author. Mailing address: Center for Research in Economics and Statistics, Finance Department, 15 Boulevard Gabriel Péri, 92245 Malakoff Cedex, France; tel: (33) 141177 727; fax: (33) 141177 666; E-mail: grassell@ensae.fr

${ }^{\dagger}$ Caisse des Dépôts et Consignations, Direction des Activités Bancaires et Financières, Paris. The views expressed in this paper are those of the authors and do not reflect those of the CDC.
} 
Afterwards, many authors have introduced a stochastic term structure for the interest rates.

On one hand, some papers like Karatzas, Lehoczky and Shreve (1987) and Karatzas (1989) do not specify the stochastic process for the interest rates, which leads to very general results. However, this general feature does not lead to explicit solutions for the optimal policies.

On the other hand, papers by Bajeux-Besnainou, Jordan and Portait (1998) and Sørensen (1999) choose a Vasiček specification for the term structure. This choice permits to obtain a closed-form solution. Bajeux-Besnainou, Jordan and Portait (1998) analyse the dynamic behaviour of the solution. Sørensen (1999) concentrates on implementation issues in a discrete time setting.

In this paper, we investigate the case where interest rates follow the CoxIngersoll-Ross (hereafter called CIR) dynamics. Contrarly to the Vasiček one, the CIR specification does not permit negative interest rates. This is the main reason why Rogers (1995) recommends to choose the CIR rather than the Vasiček specification. However, the CIR specification is less easy to manage, since in this case the distribution of the price deflator is unknown, contrarly to the Vasiček case.

Assuming completeness of the financial market and power utility function, we obtain a closed-form solution by the use of the Cox-Huang methodology.

Cox and Huang (1989) characterize the value function of the consumptioninvestment problem :

(i) as a conditional expected value,

(ii) as the solution of a linear partial differential equation; when the stochastic processes are specified, this PDE is much simpler to solve than the highly non linear Hamilton-Jacobi-Bellman equation which comes from the usual dynamic programming method.

Moreover, Cox and Huang (1989) obtain an explicit form for the value function when all the parameters (and in particular the interest rates) are constant.

In the following, we compute the value function in a CIR framework by using the first characterization. Afterwards, we study the behaviour of the solution when time approaches to the terminal date, and we compare our optimal strategies with the ones obtained in the Vasiček framework.

The paper is organized as follows: in section 2 we define the market structure and introduce the optimization problem. In section 3, we solve explicitely the problem in the power utility case. In section 4 , we analyze the 
behaviour of the solution and compare our results with the ones obtained in the Vasiček case.

\section{The model}

In this section, we present:

(i) the financial market, that is the assets available and their dynamics,

(ii) the optimization program, that is the characteristics of the agent and his optimization criteria.

\subsection{The financial market}

The market is assumed to be arbitrage-free, complete and continuously open between 0 and $T$, where $T$ is a strictly positive real number. Randomness is described by a 2-dimensional Brownian motion $\underline{z}(t)=\left\{\left(z(t), z_{r}(t)\right)^{\prime} ; t \in\right.$ $[0, T]\}$ (hereafter ' denotes transposition) defined on a complete probability space $(\Omega, \mathcal{F}, P)$, where $P$ is the real world probability. The filtration $\mathcal{F}=$ $\left(\mathcal{F}_{t}\right)_{t \geq 0}$, represents the information structure assumed to be generated by the Brownian motion and satisfying the usual conditions. Hereafter $\mathbb{E}_{t}$ stands for $\mathbb{E}\left(. \mid \mathcal{F}_{t}\right)$, the conditional expected value under the real world probability.

We suppose that the stochastic short-term interest rate process $r_{t}$ follows the CIR dynamics:

$$
d r_{t}=\left(a-b r_{t}\right) d t-\sigma_{r} \sqrt{r_{t}} d z_{r}(t), \quad t \geq 0,
$$

where the coefficients $\sigma_{r}, a, b$ and $r_{0}$ are supposed to be strictly positive constants and $2 a \geq \sigma_{r}^{2}$, so that $P\left\{r_{t}>0, \forall t \in[0, T]\right\}=1$.

Note that, without loss of generality, we use $-\sigma_{r} \sqrt{r_{t}} d z_{r}(t)$ in equation (1) instead of the CIR notation $+\sigma_{r} \sqrt{r_{t}} d z_{r}(t)$, since this choice leads to a positive volatility term in the bond's dynamics. Indeed, in our settings, and with a bond market price of risk equal to $\lambda_{r} \sqrt{r_{t}}$, Cox, Ingersoll and Ross (1985) obtained the following results:

(Cox, Ingersoll and Ross 1985)

(i) The price at time $t$ of a zero-coupon bond with maturity $T$, denoted by $B(t, T)$, follows the stochastic differential equation

$$
\frac{d B(t, T)}{B(t, T)}=r_{t} d t+\sigma_{B}\left(T-t, r_{t}\right)\left(d z_{r}(t)+\lambda_{r} \sqrt{r_{t}} d t\right), \quad B(T, T)=1
$$


with volatility:

$$
\sigma_{B}\left(T-t, r_{t}\right)=\sigma_{r} h(T-t) \sqrt{r_{t}}
$$

where

$$
\begin{aligned}
& h(t)=\frac{2\left(e^{\delta t}-1\right)}{\delta-\left(b-\sigma_{r} \lambda_{r}\right)+e^{\delta t}\left(\delta+b-\sigma_{r} \lambda_{r}\right)}, \quad t \geq 0, \\
& \delta=\quad \sqrt{\left(b-\sigma_{r} \lambda_{r}\right)^{2}+2 \sigma_{r}^{2}},
\end{aligned}
$$

(ii) The price of the bond is given by the explicit formula

$$
B(t, T)=\exp \left\{-a \phi(T-t)-r_{t} h(T-t)\right\}
$$

where

$$
\phi(t)=-\frac{2}{\sigma_{r}^{2}} \log \left(\frac{2 \delta \exp \left(\frac{\delta+b-\sigma_{r} \lambda_{r}}{2} t\right)}{\delta-\left(b-\sigma_{r} \lambda_{r}\right)+e^{\delta t}\left(\delta+b-\sigma_{r} \lambda_{r}\right)}\right), \quad t \geq 0,
$$

Note that the function $h(t)$ is the derivative of $\phi(t)$.

We suppose that the financial market contains three assets, which can be bought or sold without incurring any restriction like short sales constraints or trading costs. With these three assets, the market is complete, which means that all risks can be replicated by a self-financing portfolio.

The first asset is the riskless asset (i.e. the cash): its price, denoted by $S_{0}(t), t \geq 0$, evolves according to:

$$
\frac{d S_{0}(t)}{S_{0}(t)}=r_{t} d t, \quad S_{0}(0)=1
$$

The second asset is a zero-coupon bond with maturity $T$ and the third asset is a stock, whose price is denoted by $S(t), t \geq 0$. The dynamics of $S(t)$ are given by:

$$
\frac{d S(t)}{S(t)}=r_{t} d t+\sigma_{1}\left(d z(t)+\lambda_{1} d t\right)+\sigma_{2} \sqrt{r_{t}}\left(d z_{r}(t)+\lambda_{r} \sqrt{r_{t}} d t\right), \quad S(0)=1,
$$

where the parameters $\left(\sigma_{1}>0, \sigma_{2}>0, \lambda_{1}, \lambda_{r}\right)$ are assumed to be constant. Remember that $z$ and $z_{r}$ are independent.

Notice that Merton (1971) considered in a constant interest rate framework only the first volatility term. As the stock prices also will be influenced by the stochastic interest rates, we introduce the term with the Brownian 
motion of the short-term interest rates with the corresponding CIR market price of risk (see Bajeux-Besnainou et al. 1998, 1999 or Sørensen 1999 in a Vasicek framework). This is equivalent to assuming that the market price of risk vector equals $\underline{\lambda}=\left(\lambda_{1}, \lambda_{r} \sqrt{r_{t}}\right)^{\prime}$. We model the volatility term associated to $z_{r}$ by analogy with the bond dynamics.

For any $t \geq 0$, let us define the process

$$
H(t)=\exp \left\{\int_{0}^{t} r_{s} d s+\int_{0}^{t} \underline{\lambda}^{\prime}\left(s, r_{s}\right) d \underline{z}(s)+\frac{1}{2} \int_{0}^{t}\left\|\underline{\lambda}\left(s, r_{s}\right)\right\|^{2} d s\right\},
$$

where $\|$.$\| denotes the Euclidean norm in \mathbb{R}^{2}$.

The process $H(t)$ is called the growth-optimum portfolio (see e.g. Merton 1992), while $H(t)^{-1}$ represents the shadow state-price process. It is well known that:

$$
B(t, T)=\mathbb{E}_{t}^{Q}\left[\exp \left\{-\int_{t}^{T} r_{s} d s\right\}\right]=\mathbb{E}_{t}\left[\frac{H(t)}{H(T)}\right],
$$

where $Q$ denotes the unique equivalent martingale measure defined by

$$
\frac{d Q}{d P}=\exp \left\{-\int_{0}^{T} \underline{\lambda}^{\prime}\left(t, r_{t}\right) d \underline{z}(t)-\frac{1}{2} \int_{0}^{T}\left\|\underline{\lambda}\left(t, r_{t}\right)\right\|^{2} d t\right\}
$$

\subsection{The optimization program}

We consider a financial agent endowed with a strictly positive initial wealth $Y_{0}$. His preferences are described by a utility function $U: \mathbb{R}_{+} \backslash\{0\} \rightarrow \mathbb{R}$, increasing, strictly concave, satisfying the Inada conditions $U^{\prime}(+\infty)=0$ and $U^{\prime}(0)=+\infty$, and $\lim _{y \rightarrow 0^{+}} U(y) \geq-\infty$.

These conditions are for example satisfied by the family of CRRA utility functions which are defined by:

$$
U(y)=\frac{y^{\gamma}}{\gamma}, \quad \gamma \in(-\infty, 1) \backslash\{0\}
$$

Denoting by $\left(1-\pi_{t}^{B}-\pi_{t}^{S}\right)$, (resp. $\pi_{t}^{B}$, resp. $\left.\pi_{t}^{S}\right)$ the proportion of wealth invested into the riskless asset, (resp. the bond, resp. the stock), the program of the agent is to maximize the expected utility of his terminal wealth under feasibility constraints, namely: 


$$
\max _{\left(\underline{\pi}_{t}\right)_{t \in[0, T)} \in \mathcal{A}} \mathbb{E} U(Y(T))
$$

where:

- the wealth process $(Y(t))_{t \in[0, T]}$ is defined by the following dynamics:

$$
\begin{aligned}
d Y(t) & =Y(t) \underline{\pi}_{t}^{\prime} \operatorname{diag}[\underline{S}(t)]^{-1} d \underline{S}(t) \\
Y(0) & =Y_{0}>0
\end{aligned}
$$

with $\underline{\pi}_{t}=\left(\left(1-\pi_{t}^{B}-\pi_{t}^{S}\right), \pi_{t}^{B}, \pi_{t}^{S}\right)^{\prime}, \underline{S}(t)=\left(S_{0}(t), B(t, T), S(t)\right)^{\prime}$, and where for all $\underline{x}=\left(x_{1}, x_{2}, x_{3}\right) \in \mathbb{R}^{3}$, diag $[\underline{x}]$ denotes the diagonal matrix whose $i-$ th $(i=1,2,3)$ diagonal element is $x_{i}$. The dynamics of the wealth process can also be written in an explicit way:

$$
\begin{aligned}
\frac{d Y(t)}{Y(t)}= & \left(1-\pi_{t}^{B}-\pi_{t}^{S}\right) \frac{d S_{0}(t)}{S_{0}(t)}+\pi_{t}^{B} \frac{d B(t, T)}{B(t, T)}+\pi_{t}^{S} \frac{d S(t)}{S(t)} \\
= & r_{t} d t+\pi_{t}^{B} \sigma_{B}\left(T-t, r_{t}\right)\left(d z_{r}(t)+\lambda_{r} \sqrt{r_{t}} d t\right)+ \\
& \pi_{t}^{S}\left[\sigma_{1}\left(d z(t)+\lambda_{1} d t\right)+\sigma_{2} \sqrt{r_{t}}\left(d z_{r}(t)+\lambda_{r} \sqrt{r_{t}} d t\right)\right] \\
Y(0)= & Y_{0}>0 .
\end{aligned}
$$

- $\mathcal{A}$ is the set of admissible controls, namely

$$
\begin{aligned}
\mathcal{A}= & \left\{\left(\underline{\pi}_{t}\right)_{t \in[0, T)}: \underline{\pi}_{t} i s \mathcal{F}_{t}-\right.\text { measurable, } \\
& \int_{0}^{T}\left(\left(Y(t) \pi_{t}^{B} h(T-t)\right)^{2}+\left(Y(t) \pi_{t}^{S}\right)^{2}\right) d t<+\infty P-\text { a.e. }, \\
& \left.Y(t) \geq 0 P-\text { a.e. } \forall t \in[0, T] \text { and } \mathbb{E} U^{-}(Y(T))<+\infty\right\},
\end{aligned}
$$

with $x^{-}=\max [-x, 0]$.

In order to solve this dynamic optimization problem (10), we apply the Cox-Huang methodology. Indeed, it is well known (Cox and Huang 1991) that (10) is equivalent to

$$
\begin{aligned}
\max _{Y \in L_{+}^{p}}(\Omega, \mathcal{F}, P) & \mathbb{E} U(Y) \\
\text { s.to }: & \text { Yisfinancedbysome } \underline{\pi}_{t} \in \mathcal{A} \\
& \mathbb{E}\left(\frac{Y}{H(T)}\right)=Y_{0},
\end{aligned}
$$

for some $p \geq 1$, where $L_{+}^{p}(\Omega, \mathcal{F}, P)$ is the space of positive $\mathcal{F}_{T}$-measurable random variables such that

$$
\mathbb{E}\left[|\cdot|^{p}\right]<\infty
$$


Under some growth assumption for the utility function and some integrability condition for the price deflator $\left(U(y)\right.$ bounded from above by $\beta_{1}+\beta_{2} y^{1-b}$ and $H(T) \in L^{p / b}(\Omega, \mathcal{F}, P)$ for some $\beta_{1} \geq 0, \beta_{2}>0$ and $\left.b \in(0,1)\right)$, Cox and Huang (1991) proved the existence of the optimal solution for (15). Notice that (15) is a static problem, which can be solved in a standard way via the Lagrangian theory. If we denote by $\bar{y}:=\inf \left\{y \in \mathbb{R}_{+}: U(y)>-\infty\right\}$, it follows that the inverse of the right derivative of the utility function, namely $I(x):=\left(U_{+}^{\prime}\right)^{-1}(x)$, maps $\left(0, U^{\prime}\left(\bar{y}^{+}\right)\right)$onto $(\bar{y},+\infty)$. Moreover, the optimal terminal wealth is given by (see Cox and Huang 1989)

$$
Y^{*}=I\left(\frac{\theta}{H(T)}\right) \quad P-\text { a.e. }
$$

where $\theta$ is the Lagrangian multiplier associated to (15). The optimal wealth at time $t \geq 0$ is associated to the following function:

$$
\begin{aligned}
F(H(t), t) & =\mathbb{E}_{t}\left[\frac{H(t)}{H(T)} I\left(\frac{\theta}{H(T)}\right)\right] \\
& =\mathbb{E}_{t}^{Q}\left[\exp \left\{-\int_{t}^{T} r_{u} d u\right\} I\left(\frac{\theta}{H(T)}\right)\right],
\end{aligned}
$$

where the parameter $\theta$ is determined by the budget constraint

$$
F(H(0), 0)=Y_{0} .
$$

However, when the interest rates follow the CIR dynamics, the distribution of the process $H(t)$ is not easy to handle, and therefore we will express (16) as a function of $H(T)$, and then determine a trading strategy which replicates this function.

\section{Explicit solution in the power utility case}

Let us consider the case of CRRA utility function defined in (9). In this case the optimal solution with non-negativity constraint on terminal wealth will have the same form as the optimal solution of the unconstrained problem. In fact, the CRRA utility functions exhibit an infinite marginal utility at zero wealth, so the non-negativity constraints never bind. From (9) we deduce

$$
I(x):=\left(U_{+}^{\prime}\right)^{-1}(x)=x^{\frac{1}{\gamma-1}}, 0<x<\infty,
$$


so that

$$
Y^{*}=\left(\frac{\theta}{H(T)}\right)^{\frac{1}{\gamma-1}} \quad P-\text { a.e. }
$$

While replacing (18) into (16), we have

$$
F(H(t), t)=\theta^{\frac{1}{\gamma-1}} H(t) \mathbb{E}_{t}\left[H(T)^{\frac{\gamma}{1-\gamma}}\right],
$$

where $\theta$ is determined by (17):

$$
\theta^{\frac{1}{\gamma-1}} \mathbb{E}\left[H(T)^{\frac{\gamma}{1-\gamma}}\right]=Y_{0},
$$

that is

$$
\theta=\left(\frac{\mathbb{E}\left[H(T)^{\frac{\gamma}{1-\gamma}}\right]}{Y_{0}}\right)^{1-\gamma}
$$

In fact, to compute (16), we need an expression for

$$
\mathbb{E}_{t}\left[\left(\frac{H(T)}{H(t)}\right)^{\frac{\gamma}{1-\gamma}}\right]
$$

which is the aim of the following technical lemma:

Suppose that $c$ is a real number such that

$$
c\left(1+\frac{\lambda_{r}^{2}}{2}-\frac{\lambda_{r} b}{\sigma_{r}}\right) \leq \frac{b^{2}}{2 \sigma_{r}^{2}},
$$

then there exist two deterministic functions $k_{1}(t, c), k_{2}(t, c)$ such that

$$
\mathbb{E}_{t}\left[\left(\frac{H(T)}{H(t)}\right)^{c}\right]=k_{1}(T-t, c) \exp \left\{-r_{t} k_{2}(T-t, c)\right\}
$$

For $c=0$ the statement is obvious, so we only deal with the case $c \neq 0$. From (7) it turns out that

$$
\begin{aligned}
\mathbb{E}_{t}\left[\left(\frac{H(T)}{H(t)}\right)^{c}\right]= & \mathbb{E}_{t} \exp \left\{c \int_{t}^{T}\left(r_{s}+\frac{1}{2}\left(\lambda_{1}^{2}+\lambda_{r} r_{s}\right)\right) d s+c \int_{t}^{T} \lambda_{1} d z(s)+\right. \\
& \left.+c \int_{t}^{T} \lambda_{r} \sqrt{r_{s}} d z_{r}(s)\right\}
\end{aligned}
$$


Using (1), we have that

$$
-\int_{t}^{T} \lambda_{r} \sqrt{r_{s}} d z_{r}(s)=\frac{\lambda_{r}}{\sigma_{r}}\left(r_{T}-r_{t}-a(T-t)+b \int_{t}^{T} r_{s} d s\right),
$$

so, thanks to the independency of $z(t)$ and $z_{r}(t)$, we obtain

$$
\mathbb{E}_{t}\left[\left(\frac{H(T)}{H(t)}\right)^{c}\right]=f\left(T-t, r_{t}, c\right) \cdot \mathbb{E}_{t} \exp \left\{-\eta r_{T}-\mu \int_{t}^{T} r_{s} d s\right\}
$$

where

$$
\begin{aligned}
f\left(T-t, r_{t}, c\right) & =\exp c\left\{(T-t)\left(c \frac{\lambda_{1}^{2}}{2}+\frac{\lambda_{1}^{2}}{2}+\frac{\lambda_{r} a}{\sigma_{r}}\right)+\frac{\lambda_{r}}{\sigma_{r}} r_{t}\right\}, \\
\mu & =-c\left(1+\frac{\lambda_{r}^{2}}{2}-\frac{\lambda_{r} b}{\sigma_{r}}\right) \\
\eta & =c \frac{\lambda_{r}}{\sigma_{r}}
\end{aligned}
$$

Applying to (21) the formula of Pitman and Yor (1982) we find that:

$$
\mathbb{E}_{t}\left[\left(\frac{H(T)}{H(t)}\right)^{c}\right]=f\left(T-t, r_{t}, c\right) \exp \left\{-a \phi_{\eta, \mu}(T-t)-r_{t} \psi_{\eta, \mu}(T-t)\right\}
$$

with

$$
\begin{aligned}
& \phi_{\eta, \mu}(t)=-\frac{2}{\sigma_{r}^{2}} \log \left(\frac{2 \alpha e^{\frac{(\alpha+b) t}{2}}}{\sigma_{r}^{2} \eta\left(e^{\alpha t}-1\right)+\alpha-b+e^{\alpha t}(\alpha+b)}\right), \\
& \phi_{\eta, \mu}^{\prime}(t)=\psi_{\eta, \mu}(t)=\frac{\eta\left(\alpha+b+e^{\alpha t}(\alpha-b)\right)+2 \mu\left(e^{\alpha t}-1\right)}{\sigma_{r}^{2} \eta\left(e^{\alpha t}-1\right)+\alpha-b+e^{\alpha t}(\alpha+b)},
\end{aligned}
$$

and $\alpha=\sqrt{b^{2}+2 \sigma_{r}^{2} \mu}$, which is well defined thanks to (19). Equations (25) and (22) then lead to the result, with

$$
\begin{gathered}
k_{1}(t, c)=\exp \left\{c\left(c \frac{\lambda_{1}^{2}}{2}+\frac{\lambda_{1}^{2}}{2}+\frac{\lambda_{r} a}{\sigma_{r}}\right) t-a \phi_{\eta, \mu}(t)\right\}, \\
k_{2}(t, c)=-\frac{\lambda_{r}}{\sigma_{r}} c+\psi_{\eta, \mu}(t) .
\end{gathered}
$$

Remark 1. The technical hypothesis (19) allows us to apply the formula (25) in a slightly different form with respect to the one established e.g. in 
Lamberton and Lapeyre (1991), chapter 6. In fact, there exists a constant $M$ such that the Laplace transform

$$
L\left(t, r_{t}, \beta\right)=\mathbb{E}_{t} \exp \left\{-\alpha r_{T}-\beta \int_{t}^{T} r_{s} d s\right\}
$$

exists for $-M<\beta<0$.

Remark 2. It turns out that (28) can be written as

$$
k_{2}(t, c)=-c \frac{\left(e^{\alpha t}-1\right)\left(2+\lambda_{r}^{2}(1+c)\right)}{\alpha-b-c \lambda_{r} \sigma_{r}+e^{\alpha t}\left(\alpha+b+c \lambda_{r} \sigma_{r}\right)} .
$$

Now, since from (20) with $c=-1$ we obtain (8), and then (5), it is possible to see the link among the functions $\phi(t), h(t), k_{1}(t, c)$ and $k_{2}(t, c)$. More precisely, from (27), (30) and (4) it follows that

$$
\begin{aligned}
& k_{1}(t,-1)=\exp \{-a \phi(t)\}, \\
& k_{2}(t,-1)=h(t) .
\end{aligned}
$$

Equipped with (20), we are now able to solve explicitly the investment problem. The solution will be given in two steps: in the first one we will compute the trading strategy which replicates the portfolio $H(t)$ in terms of the basic securities $\left(S_{0}(t), B(t, T), S(t)\right)$, afterwards we will find a strategy in terms of $H(t)$ which replicates the function $F(H(t), t)$, that is the current wealth $Y(t)$. We will denote by $\left(\underline{\pi}_{t}^{H}\right)_{t \in[0, T)}=\left(\left(1-\pi_{t}^{H B}-\pi_{t}^{H S}\right), \pi_{t}^{H B}, \pi_{t}^{H S}\right)$, $t \geq 0$ the proportions invested at time $t$ into resp. $S_{0}(t), B(t, T)$ and $S(t)$ necessary to duplicate the self-financing portfolio $H(t)$.

The strategy $\left(\underline{\pi}_{t}^{H}\right)_{t \in[0, T)}$ is given by

$$
\left(\pi_{t}^{H B}, \pi_{t}^{H S}\right)=\left(\frac{\sigma_{1} \lambda_{r}-\sigma_{2} \lambda_{1}}{\sigma_{1} \sigma_{r} h(T-t)}, \frac{\lambda_{1}}{\sigma_{1}}\right)
$$

where the function $h(T-t)$ is defined by (4).

Following e.g. Karatzas (1989), the weights of the strategy are given by

$$
\left(\begin{array}{l}
\pi_{t}^{H B} \\
\pi_{t}^{H S}
\end{array}\right)=\left[\sigma\left(t, r_{t}\right)^{\prime}\right]^{-1} \underline{\lambda}\left(t, r_{t}\right)
$$


where $\sigma\left(t, r_{t}\right)$ is the volatility matix given by

$$
\sigma\left(t, r_{t}\right)=\left(\begin{array}{ll}
0 & \sigma_{B}\left(T-t, r_{t}\right) \\
\sigma_{1} & \sigma_{2} \sqrt{r_{t}}
\end{array}\right)
$$

Then (31) follows from (3) and the definition of the market price of risk $\underline{\lambda}$. Finally, it is easy to check that $\left(\underline{\pi}_{t}^{H}\right)_{t \in[0, T)}$ is admissible.

Notice that (31) is the solution of (10) where

$$
U(y)=\log (y)
$$

and for this reason the process $H(t)$ is also called the logarithmic portfolio (see e.g. Long 1990).

Under the hypotheses of Lemma 2, the trading strategy which replicates $F(H(t), t) P-$ a.e. consists in a linear combination of the cash $S_{0}(t)$, the zero-coupon bond $B(t, T)$ and the portfolio $H(t)$, with weights resp.

$$
\begin{aligned}
& \pi_{t}^{Y S_{0}}=1-\pi_{t}^{Y B}-\pi_{t}^{Y H}, \\
& \pi_{t}^{Y B}=\frac{k_{2}\left(T-t, \frac{\gamma}{1-\gamma}\right)}{h(T-t)}, \\
& \pi_{t}^{Y H}=\frac{1}{1-\gamma},
\end{aligned}
$$

or equivalently in a combination of the cash $S_{0}(t)$, the bond $B(t, T)$ and the stock $S(t)$ with weights resp.

$$
\begin{aligned}
& \pi_{t}^{S_{0}}=1-\pi_{t}^{B}-\pi_{t}^{S} \\
& \pi_{t}^{B}=\frac{k_{2}\left(T-t, \frac{\gamma}{1-\gamma}\right)}{h(T-t)}+\frac{1}{1-\gamma} \frac{\sigma_{1} \lambda_{r}-\sigma_{2} \lambda_{1}}{\sigma_{1} \sigma_{r} h(T-t)} \\
& \pi_{t}^{S}=\frac{1}{1-\gamma} \frac{\lambda_{1}}{\sigma_{1}}
\end{aligned}
$$

From (16) and (18), we have to find the trading strategy that replicates $F(H(t), t)$, where

$$
\begin{aligned}
F(H(t), t) & =\theta^{\frac{1}{\gamma-1}} H(t) \mathbb{E}_{t}\left[H(T)^{\frac{\gamma}{1-\gamma}}\right] \\
& =\theta^{\frac{1}{\gamma-1}} H(t)^{\frac{1}{1-\gamma}} \mathbb{E}_{t}\left[\left(\frac{H(T)}{H(t)}\right)^{\frac{\gamma}{1-\gamma}}\right] .
\end{aligned}
$$

We apply the previous lemma with $c=\frac{\gamma}{1-\gamma}$ :

$$
F(H(t), t)=\left(\frac{H(t)}{\theta}\right)^{\frac{1}{1-\gamma}} k_{1}\left(T-t, \frac{\gamma}{1-\gamma}\right) \exp \left\{-r_{t} k_{2}\left(T-t, \frac{\gamma}{1-\gamma}\right)\right\}
$$


By applying the Itô formula to $F(H(t), t)$ and denoting by [.] $d t$ the deterministic factors, we have

$$
\begin{aligned}
\frac{d F(H(t), t)}{F(H(t), t)} & =\frac{1}{1-\gamma} \frac{d H(t)}{H(t)}-k_{2}\left(T-t, \frac{\gamma}{1-\gamma}\right) d r_{t}+[.] d t \\
& =\frac{1}{1-\gamma} \frac{d H(t)}{H(t)}+\frac{k_{2}\left(T-t, \frac{\gamma}{1-\gamma}\right)}{h(T-t)} \frac{d B(t, T)}{B(t, T)}+[.] d t,
\end{aligned}
$$

which says that there exists a (dynamic) combination of the processes $H(t)$, $B(t, T)$ and $S_{0}(t)$ with weights resp. $\frac{1}{1-\gamma}, \frac{k_{2}\left(T-t, \frac{\gamma}{1-\gamma}\right)}{h(T-t)}$ and $1-\frac{1}{1-\gamma}-\frac{k_{2}\left(T-t, \frac{\gamma}{1-\gamma}\right)}{h(T-t)}$, which allows to replicate $F(H(t), t) P$-a.e. In fact, by arbitrage arguments, it is clear that since the diffusion terms are equal in (35), also the drift terms are equal. The second part of the statement follows directly from (31).

Remark 3. From (35), we notice that the proportions invested into $H(t)$ and $B(t, T)$ do not sum to one and are not static. This is in contrast with the case where the interest rates follow the Vasiček dynamics, where a strong two-funds separation between $H(t)$ and $B(t, T)$ holds (see Bajeux-Besnainou, Jordan and Portait 1998). However, as in the Vasiček case (see also Sørensen 1999), the limit strategy in the limit case for $\gamma \rightarrow-\infty$ is trivial, i.e. $\pi_{t}^{B}=1$ and $\pi_{t}^{S}=\pi_{t}^{S_{0}}=0$, since

$$
\lim _{\gamma \rightarrow-\infty} k_{2}\left(t, \frac{\gamma}{1-\gamma}\right)=k_{2}(t,-1)=h(t) .
$$

\section{A dynamic study}

In this section we study the behaviour of the solution when time approaches to the terminal date and we compare our results with the dynamic study done by Bajeux-Besnainou, Jordan and Portait (1998).

Bajeux-Besnainou, Jordan and Portait (1998) considered in a Vasiček framework a model based on three assets: cash, a stock and a bond with constant time to maturity. They study power utility functions (whose ArrowPratt measure of absolute risk tolerance is linear). They find that the optimal proportion of wealth invested into the risky asset turned out to be constant but that, on the other hand, a shift of wealth from bond to cash occurs when risk aversion is large, while for less risk averse agents the proportion invested into the bond increases as time passes. 
In order to compare our results with the ones obtained in the Vasiček framework, we concentrate like them on the case of a bond with constant time to maturity $K>0$, which we denote by $B_{K}(t)$. In fact, the introduction of this bond simplifies the calculations as it is difficult to check whether the proportion $\pi_{t}^{B}$ in (34) is a decreasing function of time. We therefore rewrite (34) in terms of $S(t), B_{K}(t)$ and $S_{0}(t)$. The dynamics of $B_{K}(t)$ are given by

$$
\frac{d B_{K}(t)}{B_{K}(t)}=r_{t} d t+\sigma_{K}\left(d z_{r}(t)+\lambda_{r} \sqrt{r_{t}} d t\right), \quad B_{K}(t+K)=1,
$$

where

$$
\sigma_{K}=\sigma_{r} \sqrt{r_{t}} h(K)
$$

From (2) we can deduce the strategy which replicates $B_{K}(t)$ :

$$
\begin{aligned}
\frac{d B(t, T)}{B(t, T)} & =r_{t} d t+\sigma_{B}\left(T-t, r_{t}\right)\left(d z_{r}(t)+\lambda_{r} \sqrt{r_{t}} d t\right) \\
& =r_{t} d t+\frac{\sigma_{B}\left(T-t, r_{t}\right)}{\sigma_{K}}\left(\frac{d B_{K}(t)}{B_{K}(t)}-r_{t} d t\right) \\
& =\left(1-\frac{\sigma_{B}\left(T-t, r_{t}\right)}{\sigma_{K}}\right) \frac{d S_{0}(t)}{S_{0}(t)}+\frac{\sigma_{B}\left(T-t, r_{t}\right)}{\sigma_{K}} \frac{d B_{K}(t)}{B_{K}(t)}
\end{aligned}
$$

which then consists in investing a proportion $\frac{\sigma_{B}\left(T-t, r_{t}\right)}{\sigma_{K}}$ into $B_{K}(t)$ and $\left(1-\frac{\sigma_{B}\left(T-t, r_{t}\right)}{\sigma_{K}}\right)$ into cash.

The trading strategy which replicates $F(H(t), t) P$ - a.e. consists in a linear combination of cash $S_{0}(t)$, the bond $B_{K}(t)$ and the stock $S(t)$ with weights resp.

$$
\begin{aligned}
& \pi_{t}^{Y S_{0}}=1-\pi_{t}^{Y K}-\pi_{t}^{Y S} \\
& \pi_{t}^{Y K}=\frac{k_{2}\left(T-t, \frac{\gamma}{1-\gamma}\right)}{h(K)}+\frac{1}{1-\gamma} \frac{\sigma_{1} \lambda_{r}-\sigma_{2} \lambda_{1}}{\sigma_{1} \sigma_{r} h(K)}, \\
& \pi_{t}^{Y S}=\frac{1}{1-\gamma} \frac{\lambda_{1}}{\sigma_{1}} .
\end{aligned}
$$

Immediate from (34) and (37).

The proportion of wealth invested into the bond $B_{K}(t)$ is an increasing function of time if and only if $\gamma>0$.

From (38) it results

$$
\begin{aligned}
\frac{d}{d t} \pi_{t}^{Y K} \geq 0 & \Longleftrightarrow \frac{d}{d t} k_{2}\left(T-t, \frac{\gamma}{1-\gamma}\right) \geq 0 \\
& \Longleftrightarrow \frac{d}{d t} k_{2}\left(t, \frac{\gamma}{1-\gamma}\right) \leq 0 \\
& \Longleftrightarrow \psi_{\eta, \mu}^{\prime}(t) \leq 0 .
\end{aligned}
$$


Since $\psi_{\eta, \mu}^{\prime}(t) \leq 0$ if and only if $\psi_{\eta, \mu}^{\prime}\left(\frac{\ln t}{\alpha}\right) \leq 0$, we make the change of variable $z:=\exp \alpha t$ in (26), which becomes a ratio of two linear functions of $z$. By imposing $\psi_{\eta, \mu}^{\prime}(z) \leq 0$, we find the following relation:

$$
\sigma_{r}^{2} \eta^{2}+2 \eta b \geq 2 \mu .
$$

Now, by replacing (23) and (24)

$$
\begin{aligned}
\frac{d}{d t} \pi_{t}^{K} & \geq 0 \Longleftrightarrow c^{2} \lambda_{r}^{2}+2 c b \frac{\lambda_{r}}{\sigma_{r}} \geq-2 c\left(1+\frac{\lambda_{r}^{2}}{2}-\frac{\lambda_{r} b}{\sigma_{r}}\right) \\
& \Longleftrightarrow c\left((c+1) \lambda_{r}^{2}+2\right) \geq 0,
\end{aligned}
$$

and we obtain the statement, since $c=\frac{\gamma}{1-\gamma}$.

When risk aversion is high, that is for $\gamma<0$, the solution suggests to shift the proportion of the wealth invested into the bond $B_{K}(t)$ to cash as time passes, while the proportion invested into $S(t)$ remains constant (this due to the CRRA utility function). For $\gamma \in(0,1)$, the proportion invested into the bond $B_{K}(t)$ increases: since $S_{0}(t)$ is itself a risky asset in a stochastic interest rates framework, it is quite natural to prefer the risk premium of the bond $B_{K}(t)$ in order to improve the performance of the cash. This conclusion is perfectly analogous to the one obtained in the Vasiček framework.

Acknowledgments. The authors would like to thank Nizar Touzi, Huyen Pham and Marc Yor for their helpful comments.

\section{References}

[1] Bajeux-Besnainou, I., J. V. Jordan and R. Portait (1998) "Dynamic Asset Allocation for Stocks, Bonds and Cash over Long Horizons", presented at the 1998 Southern Finance Association conference and at the October 1998 Bachelier Seminar in Paris.

[2] Bajeux-Besnainou, I., J. V. Jordan and R. Portait (1999) "On the BondStock Asset Allocation Puzzle", presented at the 1999 Eastern Finance Association conference.

[3] Cox, J., and C. F. Huang (1989) "Optimal Consumption and Portfolio Policies When Asset Prices Follow A Diffusion Process", Journal of Economic Theory, 49, 33-83. 
[4] Cox, J. and C. F. Huang (1991) "A Variational Problem arising in Financial Economics", Journal of Mathematical Economics, 20, 465-487.

[5] Cox, J. C., Ingersoll J. E. and S. A. Ross (1985) "A Theory of the Term Structure of Interest Rates", Econometrica, 53, 385-408.

[6] Karatzas, I. (1989) "Optimization problems in the theory of continuous trading". SIAM. Journal of Control and Optimization, 27, 1221-1259.

[7] Karatzas, I., J. P. Lehoczky and S. Shreve (1987) "Optimal Portfolio and Consumption Decisions for a "Small Investor" on a Finite Horizon". SIAM. Journal of Control and Optimization, 25, 1557-1586.

[8] Lamberton, D. and B. Lapeyre (1991), "Introduction au calcul stochastique appliqué à la finance", Mathématiques et Applications, Ellipses, Paris.

[9] Long, J. B. (1990) "The Numeraire Portfolio", Journal of financial Economics, 26, 29-69.

[10] Merton, R. C. (1971) "Optimum comsumption and portfolio rules in a continuous-time case", Journal of Economic Theory, 3, 373-413. Erratum: ibid. 6 (1973), 213-214.

[11] Merton, R. (1992) "Continuous Time Finance", Blackwell.

[12] Pitman, J. and M. Yor (1982) "A Decomposition of Bessel Bridges", $Z$. Wahrscheinlichkeistheorie verw. Gebiete, Springer, 59, 425-457.

[13] Rogers, L. C. G. (1995) "Which model for term-structure of interest rates should one use?" in IMA Vol. 65: Mathematical Finance, M. H. A. Davis et al., eds. Springer, Berlin Heidelberg New York, pp. 93-116.

[14] Sørensen, C. (1999) "Dynamic Asset Allocation and Fixed Income Management", Journal of Financial and Quantitative Analysis, 34, 513-531. 